in vivo $33: 31-40(2019)$

doi:10.21873/invivo.11435

\title{
Gestational Diabetes and T-cell (Th1/Th2/Th17/Treg) Immune Profile
}

\author{
EMMANOUIL SIFNAIOS $^{1}$, GEORGE MASTORAKOS ${ }^{2}$, KATERINA PSARRA ${ }^{3}$, \\ NIKOLAOS-DIMITRIOS PANAGOPOULOS ${ }^{1}$, KONSTANTINOS PANOULIS ${ }^{1}$, \\ NIKOLAOS VITORATOS ${ }^{1}$, DEMETRIOS RIZOS ${ }^{4}$ and GEORGE CREATSAS ${ }^{1}$ \\ ${ }^{1}$ 2nd Department of Obstetrics and Gynecology, Medical School, Aretaieion University Hospital, \\ National and Kapodistrian University of Athens, Athens, Greece; \\ ${ }^{2}$ Endocrine Unit, Medical School, Aretaieion University Hospital, \\ National and Kapodistrian University of Athens, Athens, Greece; \\ ${ }^{3}$ Department of Immunology - Histocompatibility, Evangelismos Hospital, Athens, Greece; \\ ${ }^{4}$ Hormone Laboratory, Medical School, Aretaieion University Hospital, \\ National and Kapodistrian University of Athens, Athens, Greece
}

\begin{abstract}
Background/Aim: Gestational diabetes mellitus $(G D M)$ is a common pregnancy complication, characterized by insulin resistance and low-grade systemic inflammation with a pro-inflammatory immune system response. Our objective was to study the peripheral Th1, Th2, Th17 and Treg response in GDM compared to normal pregnancy. Materials and Methods: Th1, Th2, Th17 and Treg subsets was determined by flow cytometry based on staining for specific intracellular cytokines, as well as $C$-reactive protein $(C R P)$ and total IgE circulating levels. The health status of all offspring was also assessed 6 months post-delivery. Results: A total of 49 Caucasian adult pregnant women were enrolled into a GDM $(n=26)$ and Control $(n=23)$ group. At the third trimester of pregnancy, the GDM group had a higher proportion of Th2, Th17 and Treg cells compared to control. Contrary to the control group, the GDM group exhibited no significant change in the Th1/Th2/Th17/Treg profile postpartum. Furthermore, higher circulating CRP and total IgE levels were noted in the GDM group compared to controls. At the 6-month post-delivery assessment, 30.8\% of the offspring from the GDM group were found to have developed atopic dermatitis, food allergy or allergic proctocolitis
\end{abstract}

This article is freely accessible online.

Correspondence to: Dr. Emmanouil Sifnaios, 2nd Department of Obstetrics and Gynecology, National and Kapodistrian University of Athens, Medical School, Aretaieion University Hospital, 11528 Athens, Greece. Tel: +30 2108025115, e-mail: sifneosm@gmail.com

Key Words: Gestational diabetes, GDM, Th1, Th2, Th17, Treg, Th1/Th2/Th17/Treg immunologic profile, T-cells, pregnancy complications. compared to none from the control group. Conclusion: Compared to an uncomplicated pregnancy, GDM exhibits a significantly different peripheral T-cell profile at the third pregnancy trimester characterized by higher proportion of Th2, Th17 and Treg cells which persist six months post-delivery, while the increased high sensitivity CRP (hsCRP) levels stressed the low-grade inflammatory profile of this disease.

Gestational diabetes mellitus (GDM) is defined as diabetes first diagnosed during the second or third pregnancy trimester which was neither preexisting type 1 diabetes nor overt type 2 diabetes (T2DM) prior to gestation (1). Currently, GDM constitutes one of the most common complications of pregnancy and exhibits increased prevalence in many countries worldwide, ranging from $1 \%$ to $22 \%$ depending on the studied population and the applied diagnostic criteria (2-5). Moreover, GDM is associated with increased risk of complications for both the mother (e.g., increased risk for pre-eclampsia during pregnancy and for developing overt T2DM and cardiovascular disease later in life) and the fetus/offspring (e.g., increased risk for macrosomia, shoulder dystocia, and for developing T2DM, metabolic syndrome, cardiovascular and even atopic/allergic disease later in life) (6-10). Thus, GDM is now recognized as an emerging global epidemic which follows the worldwide rise in obesity prevalence and poses an increasing burden on the public healthcare systems $(4,5)$.

Recent evidence indicates that GDM is characterized not only by increased insulin resistance and glucose intolerance, but also by a state of low-grade systemic inflammation and dysregulation of the immune system which induces an imbalance between type 1 and 2 T-helper cells (Th1 and Th2, respectively) favoring pro-inflammatory responses $(11,12)$. 
Since T-helper cells constitute an integral part of the adaptive immune response and are considered to be linked to epigenetic effects, research is now also focused on studying the T-cell response in both normal and complicated pregnancy (13-15). Most of the existing studies in this field have mainly explored the related changes in the circulating levels of cytokines that reflect the Th1 [e.g., plasma levels of interferon gamma (IFN$\gamma$ ), interleukin-2 (IL-2), and tumor necrosis factor- $\alpha$ (TNF- $\alpha$ )] and Th2 (e.g., plasma levels of IL-4 and IL-13) response (11). However, additional T-cell subsets appear implicated in the regulation of the immune response in multiple disease states, including pregnancy complications $(13,15)$. Indeed, Th17 cells, which are characterized by IL-17 production, appear to play a key role in chronic inflammation and autoimmune disorders (16). Similarly, regulatory T cells (Treg cells), which are characterized by IL-10 production, represent a relatively new T-cell subset that modulates the Th1 and Th2 immune response, as well as Th17 cells (17). Notably, both these distinct T-cell subpopulations appear implicated in various pregnancy complications; hence, it is now considered that, compared to the old Th1/Th2 paradigm, the new Th1/Th2/Th17/Treg paradigm represents more precisely the underlying immunologic profile in normal and complicated pregnancy $(13,15)$.

To date, the data regarding changes in the expanded immune profile of Th1, Th2, Th17, and Treg cells in GDM are limited, with most available studies focusing only on the plasma levels of the corresponding cytokines (11). However, the latter are circulating markers of the function of these $\mathrm{T}$ cell subsets, while intracellular cytokine staining is now emerging as the only available immunological method that allows determination of antigen-specific T-cell phenotype and function (18). As such, the aim of the present study was to explore the peripheral Th1/Th2/Th17/Treg profile in GDM compared to normal pregnancy by assessing the proportion of these T-cell subsets, both at the third trimester and 6 months postpartum, via a flow cytometric method based on staining of peripheral T-cells for the corresponding specific intracellular cytokines (i.e., IFN- $\gamma$, IL-13, IL-17 and IL-10, respectively).

\section{Materials and Methods}

Study cohort and design. For the present cross-sectional study, adult ( $>18$ years old) pregnant women were recruited into the following two study groups: (i) a group of pregnant women diagnosed with GDM during the 24th-26th gestation week without any other comorbidities; and (ii) a control group of healthy pregnant women without GDM. All pregnant women were recruited from the outpatient clinic of the 2nd Department of Obstetrics \& Gynecology, Medical School, National and Kapodistrian University of Athens (Aretaieion University Hospital, Greece) at a scheduled visit between the 24th and 26th gestation week. During this visit, a 2-h $75 \mathrm{~g}$ Oral Glucose Tolerance Test (OGTT) was performed in order to screen for GDM (19). All OGTTs were performed in the morning after overnight fasting, and GDM was diagnosed when fasting plasma glucose was $\geq 92 \mathrm{mg} / \mathrm{dl}$, or 1 -h OGTT plasma glucose was $\geq 180 \mathrm{mg} / \mathrm{dl}$, or 2 -h OGTT plasma glucose was $\geq 153 \mathrm{mg} / \mathrm{dl}(1,20)$.

Only primigravida women without T2DM were considered eligible for this study. To exclude women with overt T2DM the following criteria were considered: (i) hemoglobin A1c (HbA1c) $\geq 6.5 \%$; (ii) fasting plasma glucose $\geq 126 \mathrm{mg} / \mathrm{dl}$; (iii) $2 \mathrm{~h}$ OGTT plasma glucose $\geq 200 \mathrm{mg} / \mathrm{dl}$; (iv) symptomatic women with random plasma glucose $\geq 200 \mathrm{mg} / \mathrm{dl}$ (21). In addition, the study exclusion criteria also included smoking, presence/history of other systemic/inflammatory disease (e.g., autoimmune thyroidopathies, psoriasis, atopy, asthma, rhinitis, atopic dermatitis, food allergy) and history of any prescribed medications within the last one year. Withdrawal criteria for this study included development of other complications during pregnancy, and requirement of insulin treatment or any other treatment that could impact on the study aims (e.g., corticosteroids).

All recruited women underwent a scheduled study assessment at the third trimester of pregnancy (between 28th and 34th gestation week). Study participants diagnosed with GDM had regular glucose monitoring and were well-controlled with diet. Delivery mode and birth characteristics of all offspring were recorded. All recruited women completed a second study visit 6 months post-delivery, where the health status of all offspring was also documented, including development of atopic disease such as atopic dermatitis, food allergy (e.g., allergy to milk or egg) and allergic proctocolitis.

The present study was approved by an independent ethics review committee of the Aretaieion University Hospital, Athens, Greece and was performed in accordance with the Declaration of Helsinki. All study participants provided written informed consent prior to any study activities.

Study sampling and immunologic analyses. According to the study protocol, all recruited women underwent blood sampling in the morning after overnight fasting at both scheduled study visits, namely at the third trimester of pregnancy (28th to 34th gestation week) and 6-months post-delivery. Plasma/serum samples were prepared, aliquoted and stored at $-80^{\circ} \mathrm{C}$ until assayed.

Measurements of circulating $C$-reactive protein and total IgE antibodies. C-reactive protein (CRP) circulating levels (mg/l) were measured by a high-sensitivity CRP assay (CardioPhase hsCRP, DADE Behring, Siemens, Marburg, Germany) in all samples before and after delivery.

Total IgE concentrations (IU/ml) were also measured in all samples at the two study timepoints by an enzyme allergosorbent method (Specific and Total IgE EIA kit, HYTEC, HYCOR Biomedical Ltd., Garden Grove, CA, USA), following the manufacturer's protocol.

Detection of T-lymphocyte subsets expressing specific cytokines by flow cytometry. Blood samples at the two study timepoints were also collected in lithium heparin tubes (BD Vacutainer; BD Bioscience, San Jose, CA, USA). Peripheral blood mononuclear cells (PBMCs) were separated using gradient centrifugation (Ficoll Paque; Amersham Biosciences AB, Uppsala, Sweden; 10 min $400 x \mathrm{~g}, 18^{\circ} \mathrm{C}$ ), and subsequently washed twice with phosphate buffer saline (PBS). After suspension of these cells in RPMI 1640 medium (SigmaAldrich, St. Louis, MO, USA), the following steps were followed for 
the detection of T-lymphocyte subsets expressing specific intracellular cytokines (i.e., IFN- $\gamma$, IL-13, IL-17, and IL-10):

(a) Each half of these samples was stimulated with $25 \mu \mathrm{l}$ of $25 \mathrm{ng} / \mathrm{ml}$ phorbol myristate acetate (PMA, Sigma-Aldrich) and $20 \mu \mathrm{l}$ of $1 \mu \mathrm{g} / \mathrm{ml}$ ionomycin in the presence of $20 \mu \mathrm{l}$ of protein secretion inhibitor $10 \mu \mathrm{g} / \mathrm{ml}$ brefeldin A (BFA; Sigma-Aldrich). The other half of each sample was processed only with ionomycin and BFA, as a control sample (unstimulated sample). Both samples (stimulated and unstimulated) were incubated at $37^{\circ} \mathrm{C}$ in a water bath for $4 \mathrm{~h}$.

(b) After adding $50 \mu \mathrm{l}$ of ethylenediaminetetraacetic acid (EDTA), these samples were vortexed and incubated for $15 \mathrm{~min}$ at room temperature. Immediately after this step, samples were treated with $5 \mathrm{ml}$ FACS Permeabilizing Solution (BD Biosciences, San Jose, CA, USA) diluted 1:10 with water for injection (WFI), and then vortexed and incubated for $10 \mathrm{~min}$. Subsequently, these samples were stored at $-80^{\circ} \mathrm{C}$ until further analyzed.

(c) For analysis, all stored samples (stimulated and unstimulated) were reheated in a water bath $\left(37^{\circ} \mathrm{C}\right)$ and then washed with $7 \mathrm{ml}$ CellWash with mild stirring, after being centrifuged $(250 \mathrm{x} g$ for $10 \mathrm{~min}$ ). Subsequently, the supernatant was discarded and $0.5 \mathrm{ml}$ of CellWash was added. Each of these stimulated and unstimulated samples was aliquoted into three vials $(100 \mu \mathrm{l}$ each; 3 stimulated and 3 unstimulated vials). Each of these stimulated and unstimulated $100 \mu \mathrm{l}$ samples/vials was stained with: (i) $20 \mu \mathrm{l} \gamma 2-\gamma 1-\mathrm{CD} 4$ (control) (BD Biosciences); (ii) $20 \mu \mathrm{l} \mathrm{CD69FITC} \mathrm{(BD} \mathrm{Biosciences),}$ $5 \mu \mathrm{l}$ CD4 APC (BD Biosciences), $5 \mu \mathrm{l}$ CD3 APC-Cy7 (BD Biosciences) (vial-2); and (iii) $20 \mu \mathrm{l} \mathrm{CD69FITC,} 5 \mu \mathrm{l} \mathrm{CD} 4$ APC, $5 \mu$ CD3 APC-Cy7 (vial-3) (Biolegend, San Diego, CA, USA). All these samples/vials were vortexed and incubated in the dark for $10 \mathrm{~min}$. Following washing (300 xg/5 min; twice) with CellWash $(2 \mathrm{ml})$ and after the supernatant was discarded, they were vortexed and $250 \mu \mathrm{l} \mathrm{Cytofix/Cytoperm} \mathrm{was} \mathrm{added.} \mathrm{After} \mathrm{vortexing,} \mathrm{they} \mathrm{were}$ incubated for $20 \mathrm{~min}$ at $4^{\circ} \mathrm{C}$ and then washed twice with PermWash Buffer (diluted 1:10 with WFI, $1 \mathrm{ml}$ for every vial).

(d) In the final step, $20 \mu \mathrm{l}$ IFN- $\gamma$ PE (Biolegend) and $5 \mu \mathrm{lL}-10$ PECy7 (Biolegend) were added in the second vials (vial-2) of the stimulated and unstimulated samples, while $20 \mu \mathrm{l}$ IL-13 PE and 20 $\mu \mathrm{IL}-17$ PE-Cy7 were added in the third vials (vial-3) (Biolegend). These were vortexed and then incubated in the dark for $30 \mathrm{~min}$ at $4^{\circ} \mathrm{C}$. Washing with PermWash Buffer was repeated twice and after $450 \mu$ PBS was added, all these samples were analyzed on a BD FACSCantoII flow cytometer (BD Biosciences).

At least $10^{5}$ cells were acquired, so that T-lymphocyte subsets expressing the aforementioned intracellular cytokines could be recorded. The gating for the population of T-lymphocyte subsets was set on PBMCs according to Forward and Side Scatter Characteristics and to sequential gating of $\mathrm{CD}^{+}{ }^{+} \mathrm{CD} 4{ }^{+}$cells.

Statistical analysis. For statistical testing of normality, Q-Q plots and the Kolmogorov-Smirnov test were used. Unless stated otherwise, data are presented as means and standard deviation or median and quartiles where non-normal distribution was identified. Comparisons of continuous and categorical variables between study groups were performed using t-test and chi-square test, respectively. Non-parametric tests, i.e. Mann-Whitney $U$-test and Wilcoxon's signed rank test, were performed to determine any significant differences between study groups and timepoints for each group, respectively. Spearman's rank order correlation coefficient was used to explore correlations between measured T-cell subsets at each of the two study timepoints. Results were considered statistically significant when $p<0.05$. Statistical analyses were performed using the R software (https://ftp.cc.uoc.gr/mirrors/CRAN/).

\section{Results}

Study groups. A total of 49 Caucasian adult pregnant women were enrolled into the two study groups: (i) GDM group: 26 otherwise healthy, pregnant women diagnosed with GDM; and (ii) Control group: 23 healthy pregnant women. The two study groups were matched for age $(34.0 \pm 3.7$ and $31.6 \pm 5.2$ years for the GDM and control group, respectively; $p=0.07$ ) and BMI $\left(29.5 \pm 11.8 \mathrm{~kg} / \mathrm{m}^{2}\right.$ and $26.2 \pm 8.5 \mathrm{~kg} / \mathrm{m}^{2}$ for the GDM and control group, respectively; $p=0.7)$. None of the women in the GDM study group required insulin treatment throughout the study, and all exhibited normal OGTT results post-delivery. There were no preterm deliveries in either study group, while in the GDM group 9 cases $(34.6 \%)$ of caesarian delivery were noted (not significantly different compared to controls; $p=0.20)$. No other complications during pregnancy or delivery were noted in this study.

Comparison of peripheral T-lymphocyte subsets and inflammatory/atopic circulating markers between the two study groups and timepoints. The detected T-lymphocyte $\left(\mathrm{CD}^{+} \mathrm{CD}^{+}\right)$subsets (i.e., IFN- $\gamma^{+}$, IL-13 ${ }^{+}$, IL-17 ${ }^{+}$, and IL$10^{+}$) in proportion to the lymphocyte population, and the results for circulating CRP and total IgE levels are presented in Table I (comparisons presented for each timepoint between the GDM and control group) and Table II (comparisons presented for each study group between the two study timepoints).

Regarding the Th1 response, both at the first and second study timepoints (third pregnancy trimester and 6 months postdelivery, respectively), the proportion of $\mathrm{CD} 3{ }^{+} \mathrm{CD} 4^{+} \mathrm{IFN}-\gamma^{+}$ cells showed no significant differences between the GDM and control group (Table I; $p=0.08$ and $p=0.84$, respectively; Figure 1). Between the two study timepoints, no statistically significant change was noted in the proportion of these cells in both study groups (Table II; $p=0.48$ and $p=0.16$, respectively; Figure 1).

Regarding the Th2 response, in the GDM group the proportion of IL-13 expressing cells $\left(\mathrm{CD}^{+}{ }^{+} \mathrm{CD} 4^{+} \mathrm{IL}-13^{+}\right)$was significantly higher compared to the control group at the third pregnancy trimester (Table I; first study timepoint; $p=0.001$; Figure 1). This was reversed at 6 months after delivery when the prevalence of these Th2 cells in the control group was significantly higher compared to the GDM group (Table I; second study timepoint, $p=0.001$; Figure 1). The proportion of $\mathrm{CD}^{+}{ }^{+} \mathrm{CD} 4^{+} \mathrm{IL}-13^{+}$increased significantly between the two study timepoints only in the control group (Table II; $p=0.001$; Figure 1).

The proportion of $\mathrm{CD} 3^{+} \mathrm{CD} 4^{+} \mathrm{IL}-17^{+}$cells (Th17 response) at the third pregnancy trimester was significantly 
Table I. Lymphocyte subsets $\left(\mathrm{CD}^{+} \mathrm{CD}^{+}\right)$expressing specific intracellular cytokines (cytokine $\left.{ }^{+}\right)$proportionally to the lymphocyte population, and circulating levels of C-reactive protein (CRP) and total IgE in the recruited women with gestational diabetes (GDM group) and healthy controls (Control group) at the first (third trimester: 28th to 34th gestational week) and second (6 months post-delivery) study assessment. Comparisons presented for each timepoint between the GDM and control group.

\begin{tabular}{|c|c|c|c|}
\hline & GDM group $(n=26)$ & Control group $(n=23)$ & $p$-Value \\
\hline & \multicolumn{3}{|c|}{ 1st study timepoint (28th-34th gestation week) } \\
\hline $\mathrm{CD}^{+}{ }^{+} \mathrm{CD} 4+\mathrm{IFN}-\gamma^{+}(\%)$ & $22.5(2.5-29.5)$ & $13.4(6.15-17.35)$ & 0.08 \\
\hline $\mathrm{CD}^{+}{ }^{+} \mathrm{CD} 4+\mathrm{IL}-13^{+}(\%)$ & $0.15(0.1-0.9)$ & $0(0-0.1)$ & 0.001 \\
\hline $\mathrm{CD}^{+}{ }^{+} \mathrm{CD} 4^{+} \mathrm{IL}_{-} 17^{+}(\%)$ & $0.85(0.4-1.2)$ & $0.1(0-0.4)$ & $<0.001$ \\
\hline $\mathrm{CD}{ }^{+} \mathrm{CD} 4+\mathrm{IL}-10^{+}(\%)$ & $0.2(0.1-0.3)$ & $0(0-0.1)$ & $<0.001$ \\
\hline $\mathrm{CRP}(\mathrm{mg} / \mathrm{dL})$ & $5.4(4.3-5.7)$ & $3.6(3.4-4.4)$ & $<0.001$ \\
\hline \multirow[t]{2}{*}{ Total IgE (IU/mL) } & $30.4(11.25-75.9)$ & $10(7-18.25)$ & 0.001 \\
\hline & \multicolumn{3}{|c|}{ 2nd study timepoint (6 months post-delivery) } \\
\hline $\mathrm{CD}^{+}{ }^{+} \mathrm{CD} 4+\mathrm{IFN}-\gamma^{+}(\%)$ & $18.75(11.8-22.7)$ & $20.25(0.2-27.5)$ & 0.84 \\
\hline $\mathrm{CD}^{+}{ }^{+} \mathrm{CD} 4+\mathrm{IL}-13^{+}(\%)$ & $0.1(0-0.2)$ & $0.4(0.1-0.7)$ & 0.001 \\
\hline $\mathrm{CD}^{+}{ }^{+} \mathrm{CD} 4+{ }^{+} \mathrm{L}-17^{+}(\%)$ & $0.55(0.2-1.1)$ & $0.75(0.4-1)$ & 0.82 \\
\hline $\mathrm{CD}^{+}{ }^{+} \mathrm{CD} 4+\mathrm{IL}-10^{+}(\%)$ & $0.2(0.1-0.3)$ & $0.1(0.1-0.2)$ & 0.34 \\
\hline $\mathrm{CRP}(\mathrm{mg} / \mathrm{dL})$ & $5(4.2-5.6)$ & $3.3(3.3-3.5)$ & $<0.001$ \\
\hline Total IgE (IU/mL) & $34.33(15.36-55.76)$ & $11.97(7-18.24)$ & $<0.001$ \\
\hline
\end{tabular}

Data are presented as median (interquartile range). $p$-values $<0.05$ are considered significant (Mann-Whitney $U$-test). GDM: Gestational diabetes mellitus; IFN- $\gamma$ : interferon gamma; IL-13: interleukin 13; IL-17: interleukin 17; IL-10: interleukin 10.

higher in the GDM group compared to control (Table I; first study timepoint, $p<0.001$; Figure 1). No significant difference was noted between the two groups 6 months postdelivery (Table I; second study timepoint, $p=0.82$ ), mostly due to a significant increase in the proportion of IL-17 expressing cells in the control group (Table II; $p=0.001$; Figure 1).

Regarding the Treg response, at the third pregnancy trimester the proportion of the $\mathrm{CD}^{+} \mathrm{CD} 4^{+} \mathrm{IL}-10^{+}$cells was significantly higher in GDM group compared to the control (Table I; first study timepoint, $p<0.001$; Figure 1). However, at the 6-month post-delivery study timepoint, the proportion of these Treg cells was comparable between the two study groups (Table I, $p=0.34$; Figure 1). A statistically significant increase in the proportion of the $\mathrm{CD}^{+} \mathrm{CD} 4{ }^{+} \mathrm{IL}-10^{+}$cells between the two study timepoints was noted only in the control group (Table II, $p=0.04$; Figure 1).

Finally, the GDM group exhibited statistically significant higher circulating CRP levels both at the third pregnancy trimester and 6 months post-delivery compared to controls (Table I; both $p$-values $p<0.001$ ). Between these two study timepoints, the circulating CRP levels dropped significantly only in the control group (Table II; $p=0.001$ ). In addition, total IgE plasma levels in the GDM group were significantly higher compared to controls at both study timepoints (Table I; $p=0.001$ and $p<0.001$, respectively). The circulating levels of total IgE did not change significantly in either study group between the third pregnancy trimester and the 6-month postdelivery timepoint (Table II; $p=0.89$ and $p=0.5$, respectively).
Correlations between T-lymphocyte subsets at the two study timepoints. At the third pregnancy trimester, in the GDM group a strong positive correlation was observed between $\mathrm{T}$ cells positive for IFN- $\gamma$ and IL-17 ( $\mathrm{r}=0.81, p<0.001)$, whilst a significant, but less potent, positive association was also present between IL-10 and IL-17 expressing T-cells ( $\mathrm{r}=0.471$, $p<0.01)$. At this first study timepoint, in the control group there was a strong positive correlation between T-cells expressing IL-17 and IL-10 ( $\mathrm{r}=0.854, p<0.001)$, IL-13 and IL17 $(\mathrm{r}=0.79, p<0.001)$, and IL-13 and IL-10 $(\mathrm{r}=0.744$, $p<0.001)$.

At the 6-month post-delivery study timepoint, in the GDM group a strong positive correlation was present between T-cells expressing IL-17 and IL-10 ( $\mathrm{r}=0.594, p<0.001)$, as well as between IL-17 and IFN- $\gamma$ expressing T-cells $(\mathrm{r}=0.578, p<0.001)$, while a more modest positive correlation was present between IFN- $\gamma$ and IL-10 expressing T-cells $(\mathrm{r}=0.496, p=0.004)$. At this second study timepoint, in the control group there was a strong positive correlation between T-cells expressing IFN- $\gamma$ and IL-13 ( $\mathrm{r}=0.741, p<0.001)$, IFN$\gamma$ and IL-17 $(\mathrm{r}=0.711, p<0.001)$, and IL-13 and IL-17 $(\mathrm{r}=0.703, p<0.001)$.

Offspring characteristics at 6 months postpartum. Selected study-relevant characteristics of the offspring of the women in the two study groups are presented in Table III. A significant difference was noted only regarding the documented atopic profile of the offspring. Indeed, within the first six months after delivery, eight (30.8\%) of the offspring from the GDM group had developed atopic 
Table II. Lymphocyte subsets $\left(\mathrm{CD}^{+} \mathrm{CD}^{+}\right)$expressing specific intracellular cytokines (cytokine $\left.{ }^{+}\right)$proportionally to the lymphocyte population, and circulating levels of C-reactive protein $(C R P)$ and total IgE in the recruited women with gestational diabetes (GDM group) and healthy controls (Control group) at the first (third trimester: 28th to 34th gestational week) and second (6 months post-delivery) study assessment. Comparisons presented for each study group between the two study timepoints.

\begin{tabular}{|c|c|c|c|}
\hline & $\begin{array}{c}\text { 1st study timepoint } \\
\text { (28th-34th gestation week) }\end{array}$ & $\begin{array}{l}\text { 2nd study timepoint } \\
\text { (6 months post-delivery) }\end{array}$ & $p$-Value \\
\hline \multicolumn{4}{|c|}{ GDM group $(n=26)$} \\
\hline $\mathrm{CD}^{+}{ }^{+} \mathrm{CD} 4^{+} \mathrm{IFN}-\gamma^{+}(\%)$ & $22.5(2.5-29.5)$ & $18.75(11.8-22.7)$ & 0.48 \\
\hline $\mathrm{CD}^{+}{ }^{+} \mathrm{CD} 4+\mathrm{IL}-13^{+}(\%)$ & $0.15(0.1-0.9)$ & $0.1(0-0.2)$ & 0.12 \\
\hline CD3+CD4+IL-17+ $(\%)$ & $0.85(0.4-1.2)$ & $0.55(0.2-1.1)$ & 0.58 \\
\hline $\mathrm{CD}^{+}{ }^{+} \mathrm{CD} 4+\mathrm{IL}^{-10^{+}}(\%)$ & $0.2(0.1-0.3)$ & $0.2(0.1-0.3)$ & 0.21 \\
\hline CRP (mg/dl) & $5.4(4.3-5.7)$ & $5(4.2-5.6)$ & 0.36 \\
\hline Total IgE (IU/ml) & $30.4(11.25-75.9)$ & $34.33(15.36-55.76)$ & 0.89 \\
\hline \multicolumn{4}{|c|}{ Control group $(n=23)$} \\
\hline $\mathrm{CD}{ }^{+} \mathrm{CD} 4+\mathrm{IFN}-\gamma^{+}(\%)$ & $13.4(6.15-17.35)$ & $20.25(0.2-27.5)$ & 0.16 \\
\hline $\mathrm{CD}^{+}{ }^{+} \mathrm{CD} 4+\mathrm{IL}-13^{+}(\%)$ & $0(0-0.1)$ & $0.4(0.1-0.7)$ & 0.001 \\
\hline $\mathrm{CD}^{+}{ }^{+} \mathrm{CD} 4^{+} \mathrm{IL}-17^{+}(\%)$ & $0.1(0-0.4)$ & $0.75(0.4-1)$ & 0.001 \\
\hline $\mathrm{CD}^{+}{ }^{+} \mathrm{CD} 4+\mathrm{IL}-10^{+}(\%)$ & $0(0-0.1)$ & $0.1(0.1-0.2)$ & 0.04 \\
\hline CRP (mg/dl) & $3.6(3.4-4.4)$ & $3.3(3.3-3.5)$ & 0.001 \\
\hline Total IgE (IU/ml) & $10(7-18.25)$ & $11.97(7-18.24)$ & 0.5 \\
\hline
\end{tabular}

Data are presented as median (interquartile range). $p$-Values $<0.05$ are considered significant (Wilcoxon signed ranked test). GDM: Gestational diabetes mellitus; IFN- $\gamma$ : interferon gamma; IL-13: interleukin 13; IL-17: interleukin 17; IL-10: interleukin 10.

dermatitis, food allergy or allergic proctocolitis compared to none of the offspring from the control group $(p=0.01)$.

\section{Discussion}

This study presents novel data showing that pregnant women with GDM exhibit a significantly different peripheral T-cell profile at the third pregnancy trimester compared to healthy pregnant women, which remains practically unchanged six months post-delivery. Indeed, based on staining of T-cells for specific intracellular cytokines (i.e., IFN- $\gamma$, IL-13, IL-17 and IL-10), at the third pregnancy trimester the GDM women in our study exhibited a higher proportion of Th2, Th17 and Treg cells compared to the age- and BMI-matched healthy pregnant women in the control study group. These differences between the two study groups were not evident 6 months post-delivery due to a significant increase in the proportion of these peripheral T-cell subsets in the control group once the immunomodulatory endocrine milieu of pregnancy was lifted. Interestingly, no significant changes in any of the Th1, Th2, Th17 and Treg subsets were documented in the women with GDM between the third pregnancy trimester and six months after delivery.

It is well-established that the precise regulation of the balance between Th1 and Th2 immunity is crucial in order to achieve and maintain a favorable immunologic environment for the fetal semi-allograft throughout gestation, since abnormal pro-inflammatory responses may trigger deleterious inflammatory cascades/pathways linked to pregnancy complications (e.g., pre-eclampsia and GDM) (14, 22). Thus, in order to better facilitate the normal progression of pregnancy and the survival of the fetus in the maternal uterus, during an uncomplicated pregnancy the balance between Th1 and Th2 immunity is progressively shifted towards the latter under the effects of maternal and placental hormones (e.g., cortisol, progesterone, estradiol and human chorionic gonadotropin) $(14,15,22,23)$. Late in gestation, this is followed by a progressive Th1 shift (22). Moreover, normal pregnancy is regarded as a controlled state of enhanced systemic inflammation $(24,25)$, and progressively increasing insulin resistance which peaks in the third trimester (26). In this pregnancy environment that already poses a significant metabolic and immune challenge, GDM superimposes an enhanced degree of low-grade systemic inflammation and uncompensated insulin resistance, which is considered further linked to a dysregulation of the underlying immune response $(11,12,26)$.

In the present study, based on staining for intracellular IFN- $\gamma$ and IL13, at the third pregnancy trimester the GDM pregnant women exhibited no difference in the Th1 profile, but higher proportion of Th2 cells compared to controls. This is in line with the results of a previous study showing no 


\section{IFN- $\gamma+$ T cells (Th1 Response)}

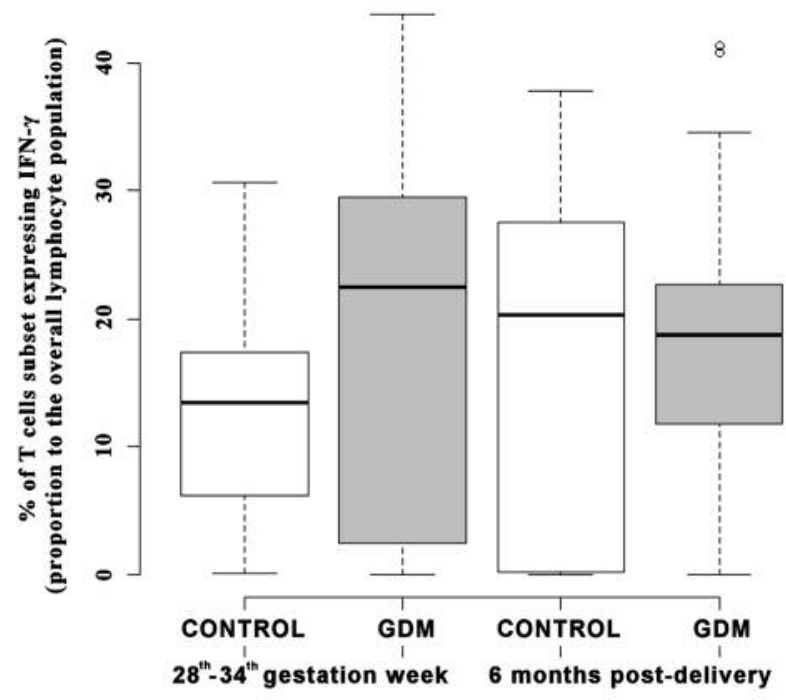

IL-17+ T cells (Th17 Response)

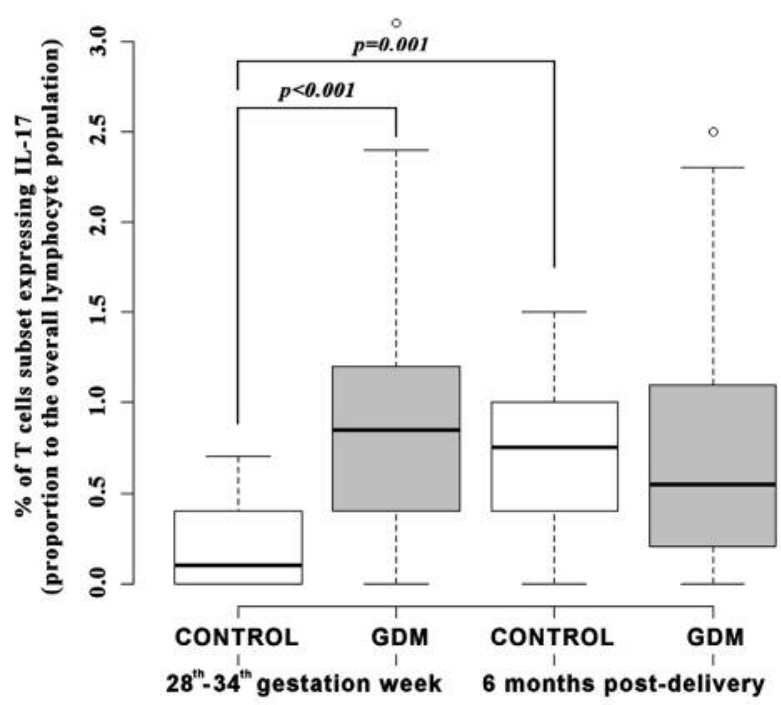

\section{IL-13+ T cells (Th2 Response)}

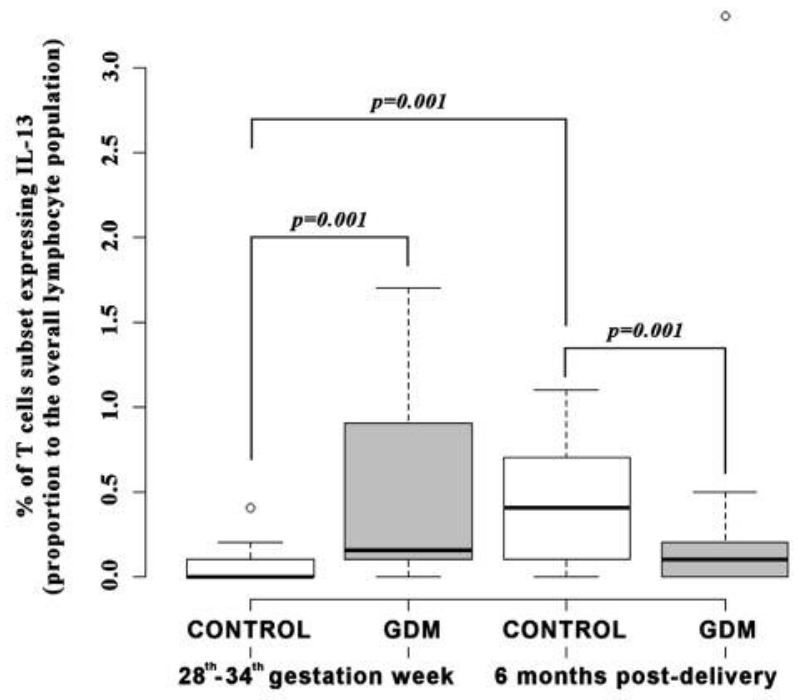

IL-10+ T cells (Treg Response)

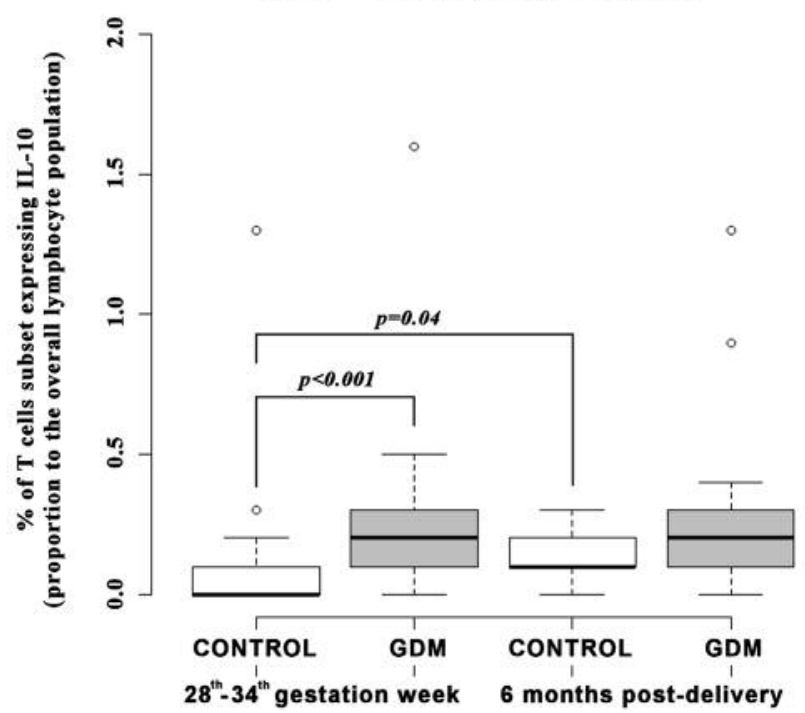

Figure 1. Box plots representing the percent proportion of $I F N-\gamma^{+}, I L-13^{+}, I L-17^{+}$and $I L-10^{+}$T-cell subpopulations among $C D 3^{+} C D 4^{+}$lymphocytes in the recruited women with gestational diabetes (GDM group) and healthy controls (Control group) at the third pregnancy trimester (28th-34th gestational week) and 6 months post-delivery. Horizontal line: median; box: interquartile range. p-Values $<0.05$ were regarded significant. IFN- $\gamma$ : Interferon gamma; IL-13: interleukin 13; IL-17: interleukin 17; IL-10: interleukin 10; Th1: T-helper 1 cells; Th2: T-helper 2 cells; Th17: T-helper 17 cells; Treg: regulatory $T$ cells.

difference in circulating IFN- $\gamma$ levels in GDM compared to non-GDM pregnancies (27). However, Ategbo et al. have reported down-regulation of Th1-related cytokines with decreased circulating IFN- $\gamma$ levels in GDM compared to non-GDM pregnant women (28). Similarly, previous studies have reported that IL-4 plasma levels (Th2-related cytokine) are either unchanged or increased in GDM compared to normal pregnancy (29-31), whilst there is also limited evidence on GDM-related changes in circulating levels of IL-13 (another key cytokine also reflecting the Th2 antiinflammatory response) (27). These non-conclusive and often conflicting findings in the existing literature illustrate 
Table III. Selected clinical, obstetrical and atopy-related data for the offspring of the recruited women with gestational diabetes (GDM group) and the healthy controls (Control group).

\begin{tabular}{lccc}
\hline Offspring characteristics & GDM group (n=26) & Control group (n=23) & $p$-Value \\
\hline Gender (male) & $12(46.2 \%)$ & $12(52.2 \%)$ & 0.78 \\
Birth Weight (kg) & $3.1(2.6-3.5)$ & $3.2(3.0-3.3)$ & 0.17 \\
Birth Length (cm) & $50(48-51)$ & $50.5(49.3-51.5)$ & 0.12 \\
Birth Head perimeter (cm) & $33.5(32.5-34.8)$ & $33.5(32.5-35)$ & 0.56 \\
Breastfeeding (months) & $4(1-5)$ & $3(1-5)$ & 0.59 \\
Introduction to formula (months) & $1(1-3)$ & $1(1-2.5)$ & 0.76 \\
Introduction to solid foods (months) & $5(4.5-5.5)$ & $5(4.5-5.5)$ & 0.6 \\
Atopic profile* & $8(30.8 \%)$ & $0(0 \%)$ & 0.01 \\
\hline
\end{tabular}

Data are expressed as median (interquartile range) for continuous variables and as number (percentage) for categorical variables. $p$-Values for comparisons between groups were given from $t$-test and chi-square test, respectively. Statistical significance was considered for $p<0.05$ for all comparisons. *Atopic profile: development of atopic dermatitis, food allergy (e.g. allergy for milk or egg), or allergic proctocolitis up to the 6month post-delivery study assessment.

the difficulty in precisely identifying the complex T-cell immune response in GDM pregnancy based on the circulating levels of the corresponding cytokines. Moreover, it should be also noted that there is a paucity of studies exploring changes in the T-cell immune response in GDM pre- and post-delivery.

In order to better understand the pathophysiology of pregnancy complications, increasing research attention is now also focused on the underlying response of Th17 cells, which are effector T-cells with a distinct lineage from Th1 and Th2 cells and are characterized by the secretion of IL-17 (13, 3234). Through IL-17 secretion, Th17 cells induce the release of pro-inflammatory mediators, as well as neutrophil recruitment, activation and migration $(15,34)$. Thus, these cells play an overall pro-inflammatory role that is vital for the defense against extracellular pathogens, but also appear implicated in the pathogenesis of chronic inflammatory and autoimmune diseases (e.g., rheumatoid arthritis, psoriasis, atopic and allergic disease) $(15,16,34)$. Of note, Th17 cells are considered to decrease in uncomplicated pregnancy compared to non-pregnant women, whilst their peripheral levels appear to stay low without significant variation during the gestation period $(15,35,36)$. The significantly increased proportion of Th17 cells at the third pregnancy trimester in the GDM women of our study compared to controls supports the notion of a more pronounced pro-inflammatory state in GDM compared to uncomplicated pregnancy. Interestingly, a previous study has reported that increased circulating IL-17 levels in GDM appear to also originate from other sources (e.g., from innate lymphoid cells 3$)(37,38)$, suggesting that the Th17 cell population in the peripheral blood of GDM women may not correlate with the IL-17 plasma levels. Thus, directly detecting the Th17 subpopulation, as in this study, may characterize more accurately the peripheral T-cell profile in GDM.
Adding to the complexity of identifying the precise peripheral profile of the T-cell immune response in GDM, it is nowadays also considered important to further detect the subset of Treg cells in the circulation $(13,15)$. These Tcells are considered essential for maintaining the desired immune homeostasis and tolerance, since they regulate and suppress the immune response $(13,15)$. In the present study the peripheral subset of IL-10-secreting Treg cells was measured, showing that at the third pregnancy trimester this T-cell subpopulation was significantly higher in the GDM pregnant women compared to controls. Taking into account the suggested link between the Th17 and Treg response (15, 34), it appears plausible that the noted higher levels of this regulatory/anti-inflammatory Treg subpopulation is linked to the concurrently higher levels of the pro-inflammatory Th17 cells in the GDM women of our study. Existing data indicate that Treg cells increase during pregnancy, peaking during the second trimester and playing a key antiinflammatory role in the required immuno-tolerance $(11,15$, 39). However, other studies have also suggested that Treg cells remain unchanged or even decrease during pregnancy $(11,40)$. Further research is clearly required to study the changes in these specific T-cell subpopulations from the first trimester of gestation in order to clarify the precise evolution of the Treg and Th17 response during GDM and uncomplicated pregnancy. This need is further highlighted by the fact that, in the existing literature unchanged, increased or even decreased IL-10 levels have been reported in the circulation of women with GDM compared to controls (11).

Circulating CRP levels were also measured in the context of our study, to exclude any underlying active inflammation and as a surrogate marker of the degree of the underlying low-grade inflammation in GDM and uncomplicated pregnancy (28). In line with the compelling evidence which 
highlights GDM as a state of heightened low-grade systemic inflammation $(11,12,26,41)$, at the third pregnancy trimester the GDM group of this study exhibited significantly higher circulating CRP levels compared to controls. At the 6-month post-delivery study timepoint when both study groups were free of the overall pro-inflammatory load of pregnancy, the circulating CRP levels remained essentially unchanged in the GDM group, whereas decreased significantly in the control group.

Finally, it should be noted that in the present study we noted no significant differences in the two study groups regarding complications (e.g., preterm delivery, caesarian delivery, macrosomia) which could be GDM-related. This may be potentially attributed to the relatively small sample size and/or the study design based on which only GDM women treated exclusively with diet and without other pregnancy complications were included in the study. However, it is noteworthy that a significant difference was documented in the atopic profile of the offspring in the present study, with $30.8 \%$ of the offspring from the GDM group developing atopic dermatitis, food allergy or allergic proctocolitis during the first six months post-delivery compared to none of the offspring from the control group. Of note, this finding potentially agrees with the significantly higher total IgE levels noted in the GDM study group compared to controls at both study timepoints. Indeed, Hicks et al. have shown that the prenatal total IgE levels of non-atopic mothers exhibit a positive association with increased risk of eczema (atopic dermatitis) in their preschool-aged (approximately up to two years old) offspring (42). Overall, the existing evidence on the relation between atopic/allergic disease and GDM is scarce. Nevertheless, there are available epidemiological data suggesting that GDM may increase the risk of early childhood atopic manifestations (e.g., atopic dermatitis) independently of fetal growth and maternal pregnancy BMI (10). Future studies are required to specifically explore whether antenatal exposure to the dysregulated metabolic and immune environment of GDM may have an epigenetic impact on the neonatal immune function and increase the risk of subsequent atopic disease.

Study limitations. The relatively small sample size of the study is a limitation. Furthermore, the lack of measurements of the corresponding cytokines in the plasma (i.e., IFN- $\gamma$, IL-13, IL-17 and IL-10 plasma levels), as well as of other circulating metabolic/ hormonal biomarkers (e.g. circulating levels of pro-inflammatory and anti-inflammatory adipokines) may constitute an additional study limitation. However, the primary objective of the present study was to directly assess the peripheral Th1/Th2/Th17/Treg profile by flow cytometry based on staining for the corresponding specific intracellular cytokines, rather than focus on related surrogate circulating biomarkers. Finally, only Caucasian pregnant women participated in this study, and, thus, the present findings cannot be directly extrapolated to pregnant women of other ethnic groups.

\section{Conclusion}

The present study adds to the existing evidence regarding the immune response in GDM by offering novel data indicating that the peripheral T-cell profile is significantly different in GDM compared to uncomplicated pregnancy at the third pregnancy trimester, remaining practically unchanged six months after delivery. Of note, the peripheral Th1/Th2/Th17/Treg profile in GDM women at the third pregnancy trimester was characterized by a higher proportion of Th17 cells (pro-inflammatory response) which was balanced with a parallel higher proportion of Th2 and Treg cells (antiinflammatory response) compared to controls. The higher circulating CRP levels both pre- and post-delivery in the GDM group of our study further supports the notion of GDM as a state of increased low-grade systemic inflammation, and could also reflect the noted enhanced pro-inflammatory immune response in this group. Finally, compared to the control group, the GDM women in our study exhibited significantly higher total IgE levels at both study timepoints, while significantly higher incidence of early atopic disease (atopic dermatitis, food allergy or allergic proctocolitis) was noted in the offspring from these GDM mothers. Future studies are required to confirm these findings in larger cohorts and study the changes in the peripheral Th1/Th2/Th17/Treg profile in GDM in conjunction with the corresponding circulating cytokines. Longer studies should also be conducted in order to assess changes of the T-cell profile from the first pregnancy trimester, and further study how these may affect the long-term risk of subsequent atopic disease in the offspring of GDM mothers.

\section{References}

1 American Diabetes Association: 2. Classification and diagnosis of diabetes: Standards of Medical Care in Diabetes - 2018. Diabetes Care 41: S13-S27, 2018.

2 Galtier F: Definition, epidemiology, risk factors. Diabetes Metab 36: 628-651, 2010

3 Jenum AK, Mørkrid K, Sletner L, Vange S, Torper JL, Nakstad B, Voldner N, Rognerud-Jensen OH, Berntsen S, Mosdøl A, Skrivarhaug T, Vardal MH, Holme I, Yajnik CS and Birkeland KI: Impact of ethnicity on gestational diabetes identified with the WHO and the modified International Association of Diabetes and Pregnancy Study Groups criteria: a population-based cohort study. Eur J Endocrinol 166: 317-324, 2012.

4 Ferrara A: Increasing prevalence of gestational diabetes mellitus: A public health perspective. Diabetes Care 30: S141-S146, 2007.

$5 \mathrm{Zhu} \mathrm{Y}$ and Zhang C: Prevalence of gestational diabetes and risk of progression to type 2 diabetes: a global perspective. Curr Diab Rep 16: 7, 2016. 
6 Kim C, Newton KM and Knopp RH: Gestational diabetes and the incidence of type 2 diabetes: a systematic review. Diabetes Care 25: 1862-1868, 2002.

7 Lekva T, Bollerslev J, Norwitz ER, Aukrust P, Henriksen T and Ueland T: Aortic stiffness and cardiovascular risk in women with previous gestational diabetes mellitus. PLoS One (SanchezMargalet V, ed.) 10: e0136892, 2015.

8 Buchanan TA, Xiang AH and Page KA: Gestational diabetes mellitus: risks and management during and after pregnancy. Nat Rev Endocrinol 8: 639-649, 2012.

9 Shah BR, Retnakaran R and Booth GL: Increased risk of cardiovascular disease in young women following gestational diabetes mellitus. Diabetes Care 31: 1668-1669, 2008.

10 Kumar R, Ouyang F, Story RE, Pongracic JA, Hong X, Wang G, Pearson C, Ortiz K, Bauchner H and Wang X: Gestational diabetes, atopic dermatitis, and allergen sensitization in early childhood. J Allergy Clin Immunol 124: 1031-1038.e1-4, 2009.

11 Lekva T, Norwitz ER, Aukrust P and Ueland T: Impact of systemic inflammation on the progression of gestational diabetes mellitus. Curr Diab Rep 16: 26, 2016.

12 Richardson AC and Carpenter MW: Inflammatory mediators in gestational diabetes mellitus. Obstet Gynecol Clin North Am 34: 213-224, 2007.

13 Saito S, Nakashima A, Shima T and Ito M: Th1/Th2/Th17 and regulatory T-Cell paradigm in pregnancy. Am J Reprod Immunol 63: 601-610, 2010.

14 Abell S, De Courten B, Boyle J and Teede H: Inflammatory and other biomarkers: role in pathophysiology and prediction of gestational diabetes mellitus. Int J Mol Sci 16: 13442-13473, 2015.

15 Polese B, Gridelet V, Araklioti E, Martens H, Perrier d'Hauterive $\mathrm{S}$ and Geenen V: The endocrine milieu and CD4 T-Lymphocyte polarization during pregnancy. Front Endocrinol 5: 106, 2014.

16 Korn T, Bettelli E, Oukka M and Kuchroo VK: IL-17 and Th17 Cells. Annu Rev Immunol 27: 485-517, 2009.

17 Fujio K, Okamura T and Yamamoto K: The family of IL-10secreting $\mathrm{CD}^{+}{ }^{+} \mathrm{T}$ cells. In: Advances in Immunology, Elsevier, pp. 99-130, 2010 .

18 Freer G: Intracellular staining and detection of cytokines by fluorescence-activated flow cytometry. In: Cytokine Bioassays. Vancurova I (ed.). New York, Springer, pp. 221-234, 2014.

19 International Association of Diabetes and Pregnancy Study Groups Consensus Panel: International Association of Diabetes and Pregnancy Study Groups Recommendations on the Diagnosis and Classification of Hyperglycemia in Pregnancy. Diabetes Care 33: 676-682, 2010.

20 National Institutes of Health Consensus Development Conference Statement: Diagnosing Gestational Diabetes Mellitus, March 4-6, 2013. Obstet Gynecol 122: 358369, 2013.

21 Basevi V, Di Mario S, Morciano C, Nonino F and Magrini N: Comment on: American Diabetes Association. Standards of Medical Care in Diabetes--2011. Diabetes Care 34: e53-e53, 2011.

22 Challis JR, Lockwood CJ, Myatt L, Norman JE, Strauss JF and Petraglia F: Inflammation and pregnancy. Reprod Sci 16: 206215, 2009.

23 Mastorakos G, Valsamakis G, Papatheodorou DC, Barlas I, Margeli A, Boutsiadis A, Kouskouni E, Vitoratos N, Papadimitriou A, Papassotiriou I and Creatsas G: The role of adipocytokines in insulin resistance in normal pregnancy: visfatin concentrations in early pregnancy predict insulin sensitivity. Clin Chem 53: 1477-1483, 2007.

24 Sargent IL, Borzychowski AM and Redman CWG: NK cells and human pregnancy - an inflammatory view. Trends Immunol 27: 399-404, 2006.

25 Rusterholz C, Hahn S and Holzgreve W: Role of placentally produced inflammatory and regulatory cytokines in pregnancy and the etiology of preeclampsia. Semin Immunopathol 29: 151$162,2007$.

26 Butte NF: Carbohydrate and lipid metabolism in pregnancy: normal compared with gestational diabetes mellitus. Am J Clin Nutr 71: 1256S-1261S, 2000.

27 Georgiou HM, Lappas M, Georgiou GM, Marita A, Bryant VJ, Hiscock R, Permezel M, Khalil Z and Rice GE: Screening for biomarkers predictive of gestational diabetes mellitus. Acta Diabetol 45: 157-165, 2008.

28 Atègbo J-M, Grissa O, Yessoufou A, Hichami A, Dramane KL, Moutairou K, Miled A, Grissa A, Jerbi M, Tabka Z and Khan NA: Modulation of adipokines and cytokines in gestational diabetes and macrosomia. J Clin Endocrinol Metab 91: 41374143, 2006

29 American Diabetes Association: Standards of Medical Care in Diabetes--2011. Diabetes Care 34: S11-S61, 2011.

30 Khan N, Yessoufou A, Kim M and Hichami A: N-3 fatty acids modulate Th1 and Th2 dichotomy in diabetic pregnancy and macrosomia. J Autoimmun 26: 268-277, 2006.

31 Yessoufou A, Hichami A, Besnard P, Moutairou K and Khan NA: Peroxisome proliferator-activated receptor $\alpha$ deficiency increases the risk of maternal abortion and neonatal mortality in murine pregnancy with or without diabetes mellitus: Modulation of T cell differentiation. Endocrinology 147: 4410-4418, 2006.

32 Park H, Li Z, Yang XO, Chang SH, Nurieva R, Wang YH, Wang Y, Hood L, Zhu Z, Tian Q and Dong C: A distinct lineage of $\mathrm{CD} 4 \mathrm{~T}$ cells regulates tissue inflammation by producing interleukin 17. Nat Immunol 6: 1133-1141, 2005.

33 Harrington LE, Hatton RD, Mangan PR, Turner H, Murphy TL, Murphy KM and Weaver CT: Interleukin 17-producing CD4+ effector T cells develop via a lineage distinct from the T helper type 1 and 2 lineages. Nat Immunol 6: 1123-1132, 2005.

34 Stockinger B, Veldhoen M and Martin B: Th17 T cells: Linking innate and adaptive immunity. Semin Immunol 19: 353-361, 2007.

35 Nakashima A, Ito M, Yoneda S, Shiozaki A, Hidaka T and Saito S: Short communication: circulating and decidual Th17 cell levels in healthy pregnancy: Th17 cells in healthy pregnancy. Am J Reprod Immunol 63: 104-109, 2009.

36 Santner-Nanan B, Peek MJ, Khanam R, Richarts L, Zhu E, Fazekas de St Groth B and Nanan R: Systemic increase in the ratio between $\mathrm{Foxp}^{+}$and $\mathrm{IL}-17$-producing $\mathrm{CD}^{+}{ }^{+} \mathrm{T}$ cells in healthy pregnancy but not in preeclampsia. J Immunol 183: 70237030, 2009.

37 Barnie PA, Lin X, Liu Y, Xu H and Su Z: IL-17 producing innate lymphoid cells 3 (ILC3) but not Th17 cells might be the potential danger factor for preeclampsia and other pregnancy associated diseases. Int J Clin Exp Pathol 8: 11100-11107, 2015.

38 Jin W and Dong C: IL-17 cytokines in immunity and inflammation. Emerg Microbes Infect 2: e60-e60, 2013.

39 Aluvihare VR, Kallikourdis M and Betz AG: Regulatory T cells mediate maternal tolerance to the fetus. Nat Immunol 5: 26671, 2004. 
40 Tilburgs T, Roelen DL, van der Mast BJ, de Groot-Swings GM, Kleijburg C, Scherjon SA and Claas FH: Evidence for a selective migration of fetus-specific $\mathrm{CD} 4{ }^{+} \mathrm{CD} 25^{\text {bright }}$ regulatory $\mathrm{T}$ cells from the peripheral blood to the decidua in human pregnancy. $\mathrm{J}$ Immunol 180: 5737-5745, 2008.

41 Leipold H, Worda C, Gruber CJ, Prikoszovich T, Wagner O and Kautzky-Willer A: Gestational diabetes mellitus is associated with increased C-reactive protein concentrations in the third but not second trimester. Eur J Clin Invest 35: 752-757, 2005.
42 Hicks WB, Nageotte CG, Wegienka G, Havstad S, Johnson CC, Ownby DR and Zoratti EM: The association of maternal prenatal $\operatorname{IgE}$ and eczema in offspring is restricted to non-atopic mothers: Maternal prenatal IgE and eczema. Pediatr Allergy Immunol 22: 684-687, 2011.

Received October 6, 2018

Revised October 23, 2018

Accepted October 24, 2018 